\title{
COVERING ALGORITHMS, CONTINUUM PERCOLATION AND THE GEOMETRY OF WIRELESS NETWORKS
}

\author{
BY LORNA BOOTH, JEHOSHUA BRUCK, \\ MASSIMO FRANCESCHETTI AND RONALD MEESTER \\ University of Utrecht, California Institute of Technology, \\ California Institute of Technology and Free University of Amsterdam
}

Continuum percolation models in which each point of a two-dimensional Poisson point process is the centre of a disc of given (or random) radius $r$, have been extensively studied. In this paper, we consider the generalization in which a deterministic algorithm (given the points of the point process) places the discs on the plane, in such a way that each disc covers at least one point of the point process and that each point is covered by at least one disc. This gives a model for wireless communication networks, which was the original motivation to study this class of problems.

We look at the percolation properties of this generalized model, showing that an unbounded connected component of discs does not exist, almost surely, for small values of the density $\lambda$ of the Poisson point process, for any covering algorithm. In general, it turns out not to be true that unbounded connected components arise when $\lambda$ is taken sufficiently high. However, we identify some large families of covering algorithms, for which such an unbounded component does arise for large values of $\lambda$.

We show how a simple scaling operation can change the percolation properties of the model, leading to the almost sure existence of an unbounded connected component for large values of $\lambda$, for any covering algorithm.

Finally, we show that a large class of covering algorithms, which arise in many practical applications, can get arbitrarily close to achieving a minimal density of covering discs. We also construct an algorithm that achieves this minimal density.

1. Introduction and motivation. Geometric covering algorithms have been extensively studied in the last 20 years, in the context of computational geometry and combinatorial optimization [see the survey by Agarwal and Sharir (1998), Section 7.1]. More recently, distributed versions of these algorithms have been proposed in the context of wireless network architectures [see Gerla and Tsai (1995)].

Communication networks are often modelled using random graphs. In such graphs vertices represent communication endpoints and edges represent two-way channels. Early work was done by Erdős and Rényi (1959, 1960, 1961a, b), but this was not suited to accurately representing networks of short-range radio transmitting stations. This motivated Gilbert (1961) to propose an alternative

Received May 2001; revised April 2002.

AMS 2000 subject classifications. 60D05, 60K35, 82B26, 82B43, 94C99.

Key words and phrases. Covering algorithms, (continuum) percolation, wireless communication networks, phase transition. 
model in which the range of the transmitters is a parameter. In his paper, he constructed a random network by considering a two-dimensional Poisson point process and joining a pair of points by an edge if discs of radius $r$ centered at those two points intersect. He was the first to introduce the concept of continuum percolation, identifying a phase transition behavior, that is, the existence of a critical value $\lambda_{c}$ for the density of the Poisson point process, at which an unbounded connected graph a.s. forms and the network can provide some long distance communication. His results were later extended, from a purely mathematical standpoint, by Hall (1985), Menshikov (1986), Roy (1990), Meester and Roy (1994) and others, leading to a theory of random coverage processes [see the books by Hall (1988) and Meester and Roy (1996)]. In a more applied framework, Gupta and Kumar $(1998,2000)$ and Penrose (1997) recently used a similar model to determine connectivity and throughput capacity of a wireless network.

In this paper we make a connection between covering algorithms and continuum percolation by considering the generalized model in which a deterministic algorithm (given the points of the point process) places the discs on the plane, in such a way that each disc covers at least one point of $X$, and each point is covered by at least one disc (see Figure 1 for a visual example).

In our model, we differentiate between base stations and clients: clients communicate between each other by connecting to base stations that forward their messages to their destinations (see Figure 2). If a client is within a given distance of a base station, it can connect to it and we say that the client is covered by the base station. Hence, the network appears as a set of circular cells (base station broadcast domains) that cover a set of points (clients). A covering algorithm decides where to place the cells, according to the distribution of the clients. The algorithm can be a distributed, self-organizing one, in a model where the entire population of clients elects "cluster heads" and divides itself into subsets that are covered by the cluster heads [see Gerla and Tsai (1995), although in that paper the base stations are chosen among the Poisson points] or a more centralized one, in a model where
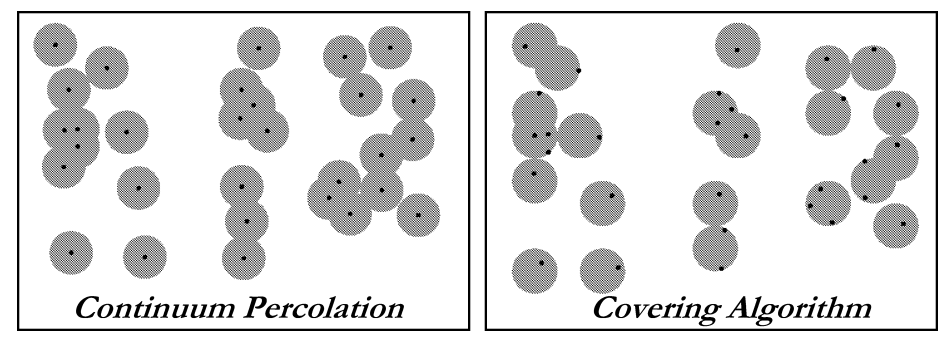

FIG. 1. Two different disc coverings of a random point process: a continuum percolation model places a disc at each random point; in our generalised model a covering algorithm places (possibly fewer) discs to cover all the points, according to a deterministic rule. We are interested in the almost sure existence of an unbounded connected component of discs, for a given density of points $\lambda$. 


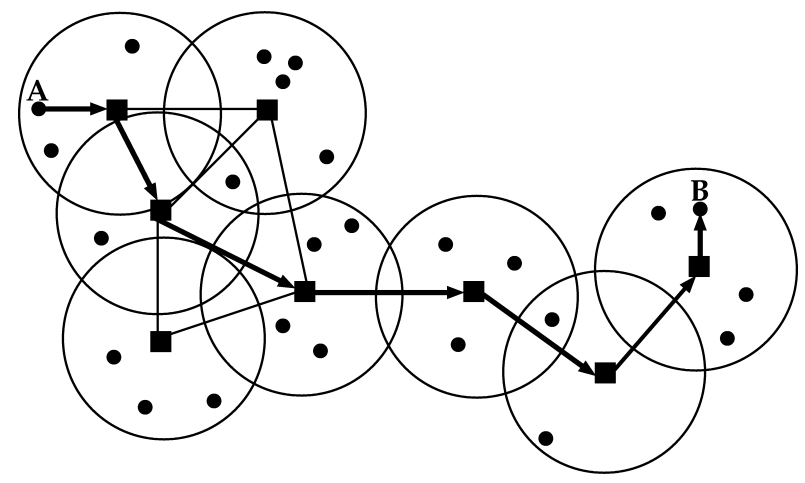

FIG. 2. The wireless backbone: a connected component of discs forms the wireless backbone of the network. If a client A wants to communicate to a client $B$, it connects to the closest base station and its message is routed through the backbone in a multi-hop fashion, until it reaches client $B$.

the clients are mobile and the base stations are static. In the latter case, the base stations could be laid on a fixed grid and the covering algorithm could determine the subset of them that need to be turned on, at any given time, to provide coverage [see Franceschetti, Cook and Bruck (2001)]. The algorithm would typically try to minimize the number of base stations that need to be turned on to cover all the clients, or, in the dynamic case, would try to minimize the base station movement needed to cover all the clients.

As in Gilbert's model, we assume a completely wireless network; hence, base stations can connect to other base stations only up to a limited distance. We assume two base stations to be connected only if the corresponding discs overlap (although we will modify this requirement later in the paper). Therefore, if two clients are in the same connected component of overlapping discs, they can communicate, because they are reached by a connected path of base stations of that component.

In percolation theory one is interested in unbounded connected components. In our setting, unbounded connected components are of interest because they represent long-range communication. The almost fully connected state of the wireless network is also interesting, and any of the proof techniques we use to prove percolation will also show that most of the clients fall inside the unbounded connected component and are therefore able to communicate, under similar conditions. In this case, a few additional "bridge" stations can be added to connect isolated components to the unbounded one and achieve the full connectivity of the network.

It may be argued that not allowing transmitters to be further than a certain distance from a client is an artificial constraint if we are interested in having longrange communication. However, we wish to see when long-range communication occurs spontaneously, given that the clients require communication with the base 
stations and the natural restriction of not placing a base station where it does not cover any client.

We informally summarize our main results next; in Section 2 we introduce some notation and definitions; Sections 3 is devoted to existence and nonexistence results for unbounded components, for different covering algorithms; Section 4 considers the effect of varying the base station communication radius; Section 5 discusses the optimality of covering algorithms; Section 6 contains some open problems.

Our results can be grouped into three categories: (non)existence of percolation for different classes of covering algorithms; scaling results; and results concerning the optimality of certain algorithms. In the following, we let $X$ be a twodimensional Poisson point process of density $\lambda$. The points of $X$ represent the clients that are covered by base stations.

In Section 3 we show that, for any algorithm, there is no percolation for $\lambda$ sufficiently small (for any algorithm). On the other hand, there are algorithms that never percolate, whatever the value of $\lambda$. Furthermore, we identify different families of covering algorithms that do form an unbounded connected component a.s. for large $\lambda$. Finally, we show that this is not generally true for shift invariant algorithms (to be defined), by constructing a shift invariant algorithm that never percolates.

In Section 4 we introduce a further extension of our model. We note that when we consider overlapping discs as connected components, then we implicitly assume, in our model of a wireless network, that the maximum radius of communication between two base stations is twice as large as the maximum radius of communication between clients and base stations. This observation leads to the natural question of what would happen if the ratio between the two radii is different from 2.

Call $r$ the radius of the discs used to cover the points of $X$, and call $R$ the maximum distance sufficient to connect disc centers. We show that if $R / r \leq 1$, then, for any grid $G$, there is a covering algorithm that places discs only at the vertices of $G$ and a.s. does not form an unbounded connected component, for any value of $\lambda$. If $1<R / r<2$, then the same holds as long as we use a grid $G$ that is dense enough. If $R / r=2$, then, for any grid $G$, any covering algorithm that places discs only at the vertices of $G$ forms a.s. an unbounded connected component for large values of $\lambda$, but if $R / r>2$, then any algorithm forms a.s. an unbounded connected component for large values of $\lambda$, even if it is not grid-based.

Note that the latter case is useful in practice, because it states that if base stations can communicate at a distance larger than twice the maximum communication distance to the clients, an unbounded connected component forms a.s. for large values of the density of the clients, regardless of the covering algorithm used to build the cellular network.

Finally, in Section 5 we show (constructively, in Theorem 5.1) that a certain class of practical algorithms can achieve densities arbitrarily close to the optimal, and we also construct an algorithm optimal in terms of a minimal density of discs. 
2. Notation and definitions. Let $\mathbb{R}^{2}$ be the Euclidean plane, let $\mathcal{B}^{2}$ be the $\sigma$-algebra of Borel sets in $\mathbb{R}^{2}$ and let $\ell(\cdot)$ be the Lebesgue measure in $\mathbb{R}^{2}$. Let $N$ be the collection of all counting measures on $\left(\mathbb{R}^{2}, \mathscr{B}^{2}\right)$, which assign finite measure to bounded Borel sets and for which the measure of a point is at most 1 . In this way, $N$ can be identified with the set of all configurations of points in $\mathbb{R}^{2}$, without limit points. Let $\mathcal{N}$ be the $\sigma$-algebra of $N$ generated by sets of the form $\{v \in N: v(A)=k\}$, for all integers $k$ and bounded Borel sets $A$. A (planar) point process $X$ is defined as a measurable mapping from a probability space $(\Omega, \mathcal{F}, P)$ into $(N, \mathcal{N})$. For $A \in \mathcal{B}^{2}$, we denote by $X(A)$ the random number of points inside $A$. In this paper, $X$ will always be a Poisson process with density $\lambda>0$. We sometimes abuse notation and write $x \in v$, for $x \in \mathbb{R}^{2}$ and $v \in N$, to express that $x$ is one of the points of $v$.

We define a shift operation $T_{t}: \mathbb{R}^{2} \rightarrow \mathbb{R}^{2}$ as a translation in $\mathbb{R}^{2}$ over the vector $t \in \mathbb{R}^{2}$, such that $T_{t}(x)=t+x$ for all $x \in \mathbb{R}^{2}$. The shift $T_{t}$ induces in a natural way a shift transformation on $N$, which we also denote by $T_{t}$. Let, for all $x \in \mathbb{R}^{2}$ and $r \geq 0, D(x, r)$ be the disc of radius $r$ centered at $x: D(x, r)=\left\{y \in \mathbb{R}^{2}\right.$ : $|y-x| \leq r\}$. A circle of radius $r$ centered at $x$ is the set $\left\{y \in \mathbb{R}^{2}:|y-x|=r\right\}$. The boundary of a set $A$ will be denoted by $\partial A$.

We call two discs $D_{i}, D_{j}$ adjacent if $D_{i} \cap D_{j} \neq \varnothing$. We write $D_{i} \leftrightarrow D_{j}$ if there exists a sequence $D_{i_{1}}, D_{i_{2}}, \ldots, D_{i_{k}}$ of discs such that $D_{i_{1}}=D_{i}, D_{i_{k}}=D_{j}$ and $D_{i_{l}}$ is adjacent to $D_{i_{l+1}}$ for $1 \leq l<k$. A (connected) component or cluster is a set $\left\{D_{i}: i \in J\right\}$ of discs which is maximal with the property that $D_{i} \leftrightarrow D_{j}$ for all $i, j \in J$. We identify a component with the set of centers of the discs in it.

We next formally define a covering algorithm: a covering algorithm $\mathcal{A}$ with discs of radius $r$ is a measurable mapping $\mathcal{A}: N \rightarrow N$ with the following properties:

1. for all $x \in \mathcal{A}(v)$ there exists $y \in v$ such that $y \in D(x, r)$;

2. for all $y \in v$ there exists $x \in \mathcal{A}(v)$ such that $y \in D(x, r)$.

We define the occupied region $C$ of $\mathcal{A}(v)$ as the union $\bigcup_{x \in \mathcal{A}(v)} D(x, r)$.

In this paper, we examine different classes of covering algorithms, which we define as follows:

GRID ALGORITHMS. Let $G \subset \mathbb{R}^{2}$ be the set of all vertices of a twodimensional lattice. A grid algorithm $\mathcal{A}$ constrains the covering discs to be centered at the vertices of $G$. That is, $x \in \mathcal{A}(v)$ implies $x \in G$. Naturally we require $G$ to be such that every point can be covered by a disc centered at some vertex of $G$.

Flat Algorithms. A flat algorithm $\mathcal{A}$ has the property that its restriction to any box of size $n \times n$ contains at most $k(n)$ discs, for some $k(n)<\infty$. Note that $k(n)<\infty$, for some $n$, immediately implies that $k(m)<\infty$, for any $m$, as we can cover an $m \times m$ square by a finite number of $n \times n$ squares. 
FINITE HORIZON ALGORITHMS. Let $B_{n}(x)$ be the box of size $n \times n$ centered at $x$, and, for all $v \in N$, let $\left.v\right|_{B_{n}(x)}$ denote the restriction of $v$ to $B_{n}(x)$. In other words, $\left.v\right|_{B_{n}(x)}$ can be identified with the set of points $\left\{v \cap B_{n}(x)\right\}$. We say that a covering algorithm $\mathcal{A}$ has finite horizon if there exists a constant $h \geq 0$ (the horizon), so that whenever $\left.v\right|_{B_{n+2 h}(x)}=\left.v^{\prime}\right|_{B_{n+2 h}(x)}$, we have $\left.\mathcal{A}(v)\right|_{B_{n}(x)}=$ $\left.\mathcal{A}\left(v^{\prime}\right)\right|_{B_{n}(x)}$, for all $n$ and $x$. In words, this means that changing $v$ outside $B_{n+2 h}(x)$ does not change the covering inside $B_{n}(x)$.

SHIFT INVARIANT ALGORITHMS. A shift invariant algorithm $\mathcal{A}$ is defined by the property that $T_{t}(\mathcal{A}(v))=\mathcal{A}\left(T_{t}(v)\right)$, for all $t$.

$n$-SQUARE ALGORITHMS. An $n$-square algorithm is obtained as follows. Partition the plane into boxes of size $n \times n$. For each such box $B_{n}$, the covering of the points inside $B_{n}$ should use the minimal number of discs possible.

Suppose now that we want to cover the points of $X$ by the covering algorithm $\mathcal{A}$; that is, we consider the measurable map $\mathcal{A} \circ X: \Omega \rightarrow N$. This Boolean model is denoted by $(X, \mathcal{A})=(X, \lambda, r, \mathcal{A})$, where $\lambda$ is the density of $X$, and $r$ is the radius of the covering discs. The law of this process is denoted by $P_{\lambda, r}$. The standard Poisson Boolean model that places a disc of radius $r$, centered at each point of $X$, is obtained when we take $\mathcal{A}$ to be the identity and is denoted by $(X, \lambda, r)$. In this model there exists $\lambda_{c}(r)$ such that for $\lambda \leq \lambda_{c}(r)$ we have no infinite cluster a.s., while for $\lambda>\lambda_{c}(r)$ there is an infinite cluster with probability 1 . We often denote $\lambda_{c}(1)$ by $\lambda_{c}$ and scaling implies that $\lambda_{c}(r)=\lambda_{c}(1) / r^{2}$ [see Meester and Roy (1996) for more details].

Next, we define the density of $(X, \mathcal{A})$. Let $N_{(X, \mathcal{A})}(n)$ be the (random) number of discs centered inside the box $B_{n}(0)$. The density of $(X, \mathcal{A})$ is given by $\lim _{n \rightarrow \infty} N_{(X, \mathcal{A})}(n) / n^{2}$, whenever this limit exists a.s. and is an a.s. constant.

Finally, we introduce one more piece of terminology. If $(X, \mathcal{A})$ contains an unbounded component of discs with positive probability, we say that $(X, \mathcal{A})$ percolates.

3. Percolation. In this long section we think of $r$ as being fixed, while $\lambda$ varies. Accordingly, we sometimes write $P_{\lambda}=P_{\lambda, r}$. We also use $P$ to mean $P_{1,1}$. The expectation under $P$ we denote by $E$.

Our first result deals with the lack of percolation for small values of $\lambda$.

THEOREM 3.1. For any covering algorithm $\mathcal{A}$, there exists a $\lambda_{0}(r)>0$ such that, for all $0<\lambda \leq \lambda_{0},(X, \lambda, r, \mathcal{A})$ does not percolate.

PROOF. Assume that, with positive probability, there is an unbounded connected component of covering discs for $(X, \lambda, r, \mathcal{A})$. Then, with positive probability, there is an unbounded connected component in the Poisson Boolean 
model $(X, \lambda, 2 r)$. That is because two intersecting covering discs in $(X, \lambda, r, \mathcal{A})$ cover points that are at a distance of at most $4 r$ to each other; and the Poisson Boolean model $(X, \lambda, 2 r)$ places discs of radius $2 r$ at each of the covered points. We then choose $\lambda_{0}=\lambda_{c} /(2 r)^{2}$, so that $(X, \lambda, 2 r)$ does not form an unbounded connected component a.s. for $\lambda \leq \lambda_{0}$.

A symmetric result to Theorem 3.1, that is, percolation for large values of $\lambda$, depends on the type of covering algorithm used:

PROPOSITION 3.2. There exists a covering algorithm $\mathcal{A}$ such that, for all $\lambda$, $(X, \lambda, r, \mathcal{A})$ does not percolate.

PROOF. The proof is constructive. Draw circles of radii $\{3 k r, k \in \mathbb{N}\}$ around the origin and notice that a.s. no Poisson point falls on any of these circles. Then cover the Poisson points with discs of radius $r$, without intersecting these circles. Notice that the circles divide the plane into finite annuli and, since each cluster of discs resides in at most one of these finite annuli, each cluster must be bounded, whatever the value of $\lambda$.

We next look at families of algorithms that do percolate for large values of $\lambda$, starting with flat algorithms. Recall that each such algorithm $\mathcal{A}$ has the property that the restriction of $\mathcal{A}$ to any box of size $n \times n$ contains at most $k=k(n)$ discs, for any value of $\lambda$. Note that this really is a weak requirement, since we can completely cover the box using at most $\alpha\lceil(n / r)\rceil^{2}$ discs, for some $\alpha \leq 1$. Any "sensible" algorithm should therefore be flat.

THEOREM 3.3. Let $\mathcal{A}$ be a flat covering algorithm. Then there exists $\lambda_{1}<\infty$ so that $(X, \lambda, r, \mathcal{A})$ percolates for all $\lambda>\lambda_{1}$.

At first sight, the statement of the theorem is counterintuitive, since we claim that we force percolation by restricting the number of discs. The point is that by restricting the number of discs (independently of $\lambda$ ), the requirement of covering all points with this restricted number of discs makes percolation unavoidable.

The following theorems are two consequences of Theorem 3.3 and its proof that are important in practice.

THEOREM 3.4. For any grid covering algorithm $\mathcal{A}$, there exists a $\lambda_{1}<\infty$ such that $(X, \lambda, r, \mathcal{A})$ percolates for all $\lambda>\lambda_{1}$.

This theorem can be proved by application of Theorem 3.3, as all grid algorithms are flat. For an elegant proof offering more insight into the structure of grid algorithms see Booth (2002) and Booth, Bruck, Franceschetti and Meester (2002). 
THEOREM 3.5. For any $n>0$ there exists a $\lambda_{n}<\infty$ such that, for any $n$-square algorithm $\mathcal{A}_{n},\left(X, \lambda, r, \mathcal{A}_{n}\right)$ percolates for all $\lambda>\lambda_{n}$.

Since an $n$-square algorithm is flat, it follows from Theorem 3.3 that, for each $n$-square algorithm, we can find such a $\lambda_{n}$. The fact that this $\lambda_{n}$ depends only on $n$ and not on the particular $n$-square algorithm we choose follows from the proof of this theorem, by noting that $\lambda_{1}$ in the statement of the theorem only depends on $k(n)$.

Another version of Theorem 3.3 requires an upper bound on the density of discs, the shift invariance of the algorithm under a pair of linearly independent shifts and the algorithm to be finite horizon.

THEOREM 3.6. Let $\mathcal{A}$ be a finite horizon covering algorithm such that a constant $\delta<\infty$ exists such that

$$
\limsup _{n \rightarrow \infty} \frac{\text { number of discs in } B_{n}}{n^{2}}<\delta \quad \text { a.s. }
$$

and which is stationary under a pair of linearly independent shifts. Then there exists $\lambda_{1}<\infty$ so that $(X, \lambda, r, \mathcal{A})$ percolates for all $\lambda>\lambda_{1}$.

Johan Segers (personal communication) has pointed out that although finite horizon algorithms of bounded density which are stationary under any pair of linearly independent vectors must percolate, for a sufficiently high density of points, there exist finite horizon covering algorithms of bounded density which are stationary under shifts of one vector and do not percolate for any $\lambda$.

Before we prove Theorems 3.3 and 3.6, we first state and prove a preliminary geometric lemma.

LEMMA 3.7. Consider a collection of discs of radius $r$, with the property that at most $k(n)<\infty$ discs intersect any box of size $n \times n$. Then there exists an $\varepsilon=\varepsilon(n, r)>0$ with the following property: if either there are at least two clusters that intersect the boundaries of both $B_{n+r}(x)$ and $B_{n+2 r}(x)$ or there is a cluster wholly contained in $B_{n+2 r}(x)$, then there is a circle of radius $\varepsilon$, contained in $B_{n+3 r}(x)$, that is not intersected by any disc.

ProOF. We write $B_{n}=B_{n}(x)$. All discs that intersect $B_{n+3 r}$ must be centered inside $B_{n+5 r}$. Therefore, at most $k=k(n+5 r)$ discs intersect $B_{n+3 r}$. Let $C$ be a component that intersects the boundaries of both $B_{n+r}$ and $B_{n+2 r}$. The number of discs in $C$ that intersect $B_{n+2 r}$ is denoted by $l$. Note that $l \leq k$.

Consider a section $A B$ of the perimeter of $C$, from the boundary of $B_{n+r}$ to the boundary of $B_{n+2 r}$, which does not intersect either of these boundaries except at its ends (see Figure 3). This section has length at least $r / 2$ and consists of parts of the boundaries of at most $l$ discs, each of which appears only once. This latter fact 


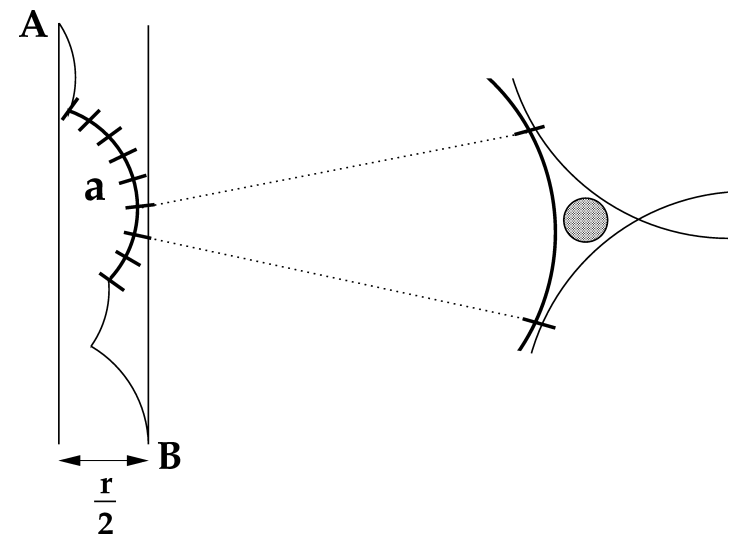

FIG. 3. Subdividing the arc: an edge AB of a component $C$ connecting the two boundaries of $B_{n+r}$ and $B_{n+2 r}$, has length at least $r / 2$. This edge contains an arc a of length at least $r / 2 l$. Arc a is divided up into $k$ sections, and by one of these we can place a small disc of radius $\varepsilon$ that is not contained in any cluster.

follows from the observation that, since the distance between the boundaries of the two boxes is only $r / 2$, any disc that contributes to an arc in $A B$ must overlap the boundary of at least one box. Moreover, note that were $A B$ to contain two disjoint arcs from the same disc, then any disc overlapping that disc, in order to make these arcs disjoint, must overlap the boundary of the box not overlapped by the first disc. Therefore these arcs would be in disjoint sections of $\partial C \cap B_{n+2 r} /\left(B_{n+r} \cup \partial B_{n+2 r}\right)$. It follows that at least one arc in $A B$ is of length at least $r / 2 l$. Call this $\operatorname{arc} a$.

Note that there are at most $k-1$ discs intersecting $B_{n+3 r}$, if we do not count the one that has $a$ as a part of its boundary, and none of them intersect $a$, except at its endpoints. If we divide $a$ into $k$ arcs of equal size, then each of these discs will be nearest to one of these smaller arcs-assign this arc to this disc. One of the smaller arcs (of size at least $r / 2 k l$ ) will, however, have no disc assigned to it. This means that the space left by discs tangent to the ends of this smaller arc cannot be covered, and we can choose $\varepsilon$ so small that a disc of radius $\varepsilon$ fits into this space (see Figure 3 ). The value of $\varepsilon$ that we have to choose only depends upon $r, n$ and $k(n)$.

The same argument applies to a component wholly contained in $B_{n+2 r}$, by considering its perimeter rather than its boundary between $B_{n+r}$ and $B_{n+2 r}$.

Proof of TheOrem 3.3. Let $t, u \in \mathbb{Z}$, and denote the box of size $n \times n$ centered at $(t n, u n)$ by $B_{n}(t n, u n)$, as before. Let $\varepsilon$ be chosen as in Lemma 3.7. We say that the vertex $(t, u)$ is a neighbor of $\left(t^{\prime}, u^{\prime}\right)$ if the boxes $B_{n}(t n, u n)$ and $B_{n}\left(t^{\prime} n, u^{\prime} n\right)$ share an edge or corner. We call a vertex $(t, u) \operatorname{good}$ if all discs of radius $\varepsilon$ contained in $B_{n+3 r}(t n, u n)$ contain at least one point of the Poisson process. Denote the event that $(t, u)$ is good by $G(t, u)$. It is clear that, when 


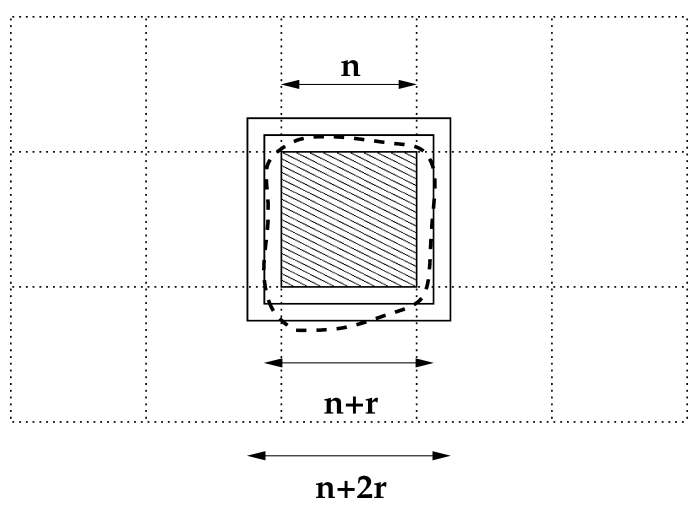

FIG. 4. A good square: there is only one component of discs (represented by the dashed line) that intersects both $B_{n+2 r}$ and $B_{n+r}$. This component must reach to within $2 \varepsilon$ of all edges of $B_{n+r}$, and will therefore intersect a component of an adjacent good square.

$\lambda \rightarrow \infty$, the probability of $G(t, u)$ converges to 1 . It is also clear that $G(t, u)$ and $G\left(t^{\prime}, u^{\prime}\right)$ are independent whenever $\max \left\{\left|t-t^{\prime}\right|,\left|u-u^{\prime}\right|\right\} \geq\left\lceil\frac{3 r}{n}\right\rceil$, for $n$ larger than $2 r$. Hence, the configuration of good sites is formed through a discrete, finite-range dependent percolation process, and it follows then from Durrett and Griffeath (1983) that, for $\lambda$ high enough, the good vertices percolate, that is, contain an infinite component of good squares with probability 1 . What does this mean for our covering? Consider a good square $B_{n}$. By Lemma 3.7 any component cannot be wholly contained in $B_{n+2 r}$; therefore, a component that covers points inside $B_{n+2 r}$ must also intersect the boundary of $B_{n+2 r}$. Also by Lemma 3.7, there can be only one component that intersects the boundaries of both $B_{n+2 r}$ and $B_{n+r}$. For $n$ larger than $2 \varepsilon$ such a component exists and must reach to within $2 \varepsilon$ of all edges of $B_{n+r}$, as, by the definition of a good square, there is no disc of radius $\varepsilon$ inside $B_{n+r}$ without any points of the Poisson process inside it (see Figure 4).

Thus, the components associated with adjacent good squares must overlap, and we must have an infinite component of discs with probability 1 .

We thank Johan Segers and the referee for the remark that this proof does not depend upon the algorithm being finite horizon.

Proof of TheOrem 3.6. The proof in this case is a little more complicated, but uses the same idea as that of Theorem 3.3. Without loss of generality assume that the algorithm be stationary under the shifts $T_{(m, 0)}$ and $T_{(0, m)}$, for some $m$. Let $h$ be the horizon of the algorithm, and assume $m>\max (1, h+5 r)$, again without loss of generality. Choose $\gamma>0$ sufficiently small so that $1-\gamma$ is strictly above the critical point for site percolation on the lattice $\{(t m, t u): t, u \in \mathbb{Z}\}$ with edges between neighboring sites. Take $n$, a multiple of $m$, so large that the probability that 
$B_{n+5 r}$ is intersected by more than $(\delta+1)(n+5 r)^{2}$ discs is less than $\gamma$, uniformly in $\lambda$. We then use $(\delta+1)(n+5 r)^{2}$ as our $k$ in the lemma and find an $\varepsilon$ such that, if we have at most $(\delta+1)(n+5 r)^{2}$ discs intersecting $B_{n+5 r}$ in the way described in the lemma, then we must have a circle of radius $\varepsilon$ empty of Poisson points.

In the proof of the theorem we then call $B_{n}$ good if $B_{n+3 r}$ has both no disc of radius $\varepsilon$ empty of Poisson points in it and $B_{n+5 r}$ contains at most $(\delta+1)(n+5 r)^{2}$ points. Other boxes are called good analogously. If $\lambda$ is high enough, these boxes percolate, and we again have an infinite component of discs.

We have seen that both finite horizon, shift invariant algorithms under a bounded density condition and grid algorithms necessarily percolate for high enough $\lambda$. It is natural to ask whether this always holds for shift invariant algorithms. It turns out that, for these algorithms, in general large values of the density $\lambda$ of the points do not guarantee the a.s. existence of an unbounded connected component. This is shown by describing a shift invariant covering algorithm that does not form an unbounded connected component for all $\lambda$ :

THEOREM 3.8. There exists a shift invariant covering algorithm $\mathcal{A}$ of all the points of $X$ by discs of radius $r$ such that, for all $\lambda,(X, \lambda, r, \mathcal{A})$ does not percolate.

The proof of Theorem 3.8 is constructive and rather technical, so we will sketch the algorithm, but omit the proof. For full details see Booth (2002) and Franceschetti (2002). The covering we describe can be seen as a shift-invariant variant of that in Theorem 3.2 and will also have a density of disc centers equal to the density of points.

SKETCH OF PROOF OF THEOREM 3.8. Without loss of generality, consider using covering discs of radius $r=1$. As the covering should be a deterministic function of the points, we must first calculate $\lambda$ in the realization of the point configuration. We do this by setting

$$
\lambda=\lim _{n \rightarrow \infty} \frac{\text { number of points in } B_{n}(0)}{n^{2}}
$$

if this limit exists and is constant, which happens with probability 1 , and otherwise we take $\lambda=1$.

We define a potential-point to be a Poisson point with at least one other point in the half-disc of radius $\frac{1}{2}$ to the right of it, and no points in the disc of radius 1 centered at it, except in the aforementioned half-disc. We then call a potential-point an $m$-point, for integer $m$, if it has its nearest neighboring point between $b_{m}$ and $b_{m+1}$ away, where $b_{1}=\frac{1}{2}, b_{2}, b_{3}, \ldots$ is a decreasing sequence of positive numbers, such that the density of $m$-points is exactly $\varepsilon 18^{-2 m}$, for some small number $\varepsilon>0$. 


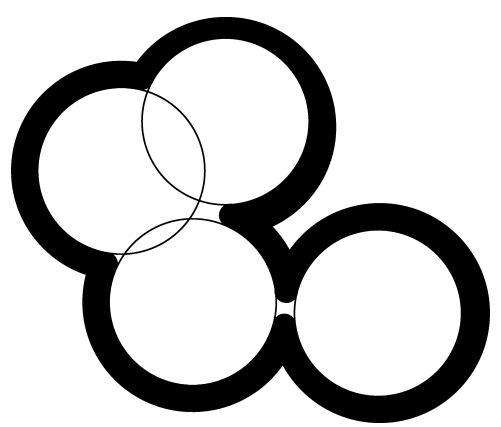

FIG. 5. Sausages: by sliding a disc of radius 2 along the boundary of some cluster, we trace a kind of sausage shape.

How small $\varepsilon$ will be required to be depends upon $\lambda$. Around each $m$-point we place a circle of radius $18^{m}$.

A theorem of Meester and Roy (1994) allows us to show that every bounded region of the plane is a.s. wholly contained in some circle. Lots more work, based upon an idea of Meester and Roy (1996) for fractal continuum percolation, shows us that each cluster of overlapping circles is finite, and moreover the maximal connected component of points strictly within distance 9 of any circle is also finite.

We construct smooth curves based upon the finite circle clusters. Consider some maximal set of circles such that, if we take the locus of points at a maximal distance of 4 from the points in the circles, then this forms a connected set, and run a disc of radius 2 around the outside of this set (see Figure 5). The disc traces out a kind of finite sausage shape around the clusters.

We take the inside edge of this sausage as our curve and note that a covering disc (of radius 1) can get arbitrarily close to any point of it without touching it. We construct these smooth curves for each set of sufficiently close clusters, noting that they surround every region, are always finite and never come within distance 8 of each other.

We finally cover our Poisson points as follows:

(a) If a point is at a distance more than 2 from every smooth curve, then we center a covering disc at the point.

(b) If a point is within distance 2 of a smooth curve, then we place a disc so that its perimeter covers the point, and so that the center of the disc is at the maximum distance away from the smooth curve. If there are a number of such possible positions, we choose the leftmost.

It immediately follows that, for any given value of $\lambda$, a.s. there is no percolation.

4. Scaling. In this section we consider an extension that is useful to model the transmission power in wireless communication networks. We look at the 

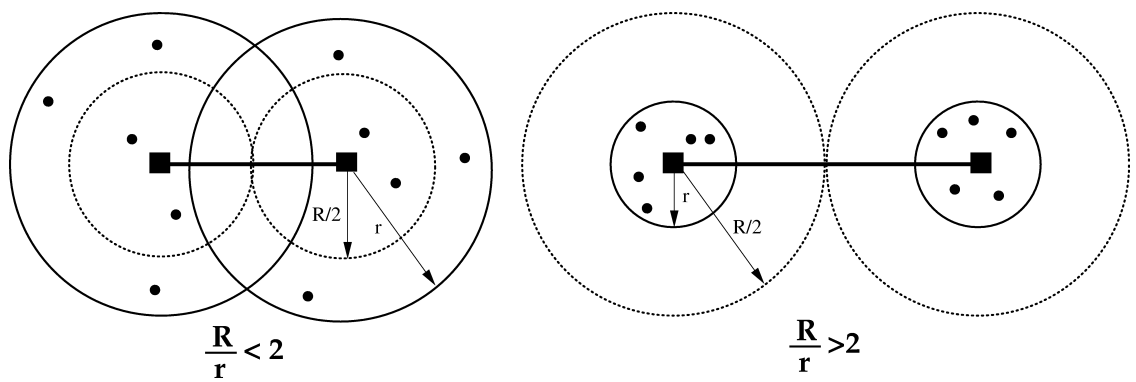

FIG. 6. Scaling: points are covered by solid line discs of radius $r$. Discs centers are considered connected if their distance is at most $R$.

percolation properties of our model, for different values of the connectivity range of the base stations and of the clients.

Let $r$ be the clients' connectivity range and let $R$ be the base stations' connectivity range. It follows that discs of radius $r$ are used to cover the points of $X$ and two disc centers are considered connected, if their distance is less than or equal to $R$ (see Figure 6). We are interested in the a.s. existence of an unbounded connected component of disc centers, for large values of the density $\lambda$ of the Poisson point process. Our result is the following.

THEOREM 4.1 (The scaling theorem). Let $G \subset \mathbb{R}^{2}$ be the set of all vertices of a square lattice in which the distance between two neighboring lattice vertices is $\delta$. Call two disc centers connected if their distance is at most $R$. We have the following cases.

Case 1 . If $\frac{R}{r} \leq 1$, then, for any $\delta>0$, there exists a grid covering algorithm $\mathcal{A}$ that places discs only at the vertices of $G$ such that, for all $\lambda,(X, \lambda, r, \mathcal{A})$ does not percolate.

Case 2. If $1<\frac{R}{r}<2$, then there exists a $\delta>0$, depending on $\frac{R}{r}$, such that there exists a grid covering algorithm A that places discs only at the vertices of $G$ and, for all $\lambda,(X, \lambda, r, \mathcal{A})$ does not percolate.

Case 3. If $\frac{R}{r}=2$, then, for any $\delta>0$, for any grid covering algorithm $\mathcal{A}$, there exists a $\lambda_{1}<\infty$ such that, for all $\lambda>\lambda_{1},(X, \lambda, r, \mathcal{A})$ percolates.

Case 4. If $\frac{R}{r}>2$, then, for any covering algorithm $\mathcal{A}$, there exists a $\lambda_{1}<\infty$ such that, for all $\lambda>\lambda_{1},(X, \lambda, r, \mathcal{A})$ percolates.

Note that Case 4 of the theorem states that, in a wireless network in which base stations can communicate at a distance larger than twice the maximum communication distance to the clients, an unbounded connected component forms a.s. for large values of the density of the clients, regardless of the covering algorithm used to build the cellular network. 


\section{PROOF OF THEOREM 4.1.}

Case 1 . We can restrict our attention to $\frac{R}{r}=1$. That is because if a grid covering algorithm does not form an unbounded connected component when $\frac{R}{r}=1$, then it does not form such a component when $\frac{R}{r}<1$ either.

Note that, for $\frac{R}{r}=1$, two disc centers are considered connected if and only if the corresponding discs of radius $r$ cover each other's centers. Moreover, in order to be able to cover all points on the plane by using only grid discs of radius $r$, the grid spacing $\delta$ must be at most $\sqrt{2} r$. We now consider all values of the grid spacing $\delta \leq \sqrt{2} r$, subdivided into intervals.

For $r<\delta \leq \sqrt{2} r$, any grid covering algorithm places discs on the plane that do not touch each other's centers.

For $\frac{r}{\sqrt{2}}<\delta \leq r$, consider the tiling of the plane depicted in the left part of Figure 7. Discs of this tiling do not cover each other's centers; therefore, any grid covering algorithm that covers all the points of $X$ using only the grid discs depicted in the left part of Figure 7 does not form an unbounded connected component, a.s., for any value of $\lambda$.

For $r / 2<\delta \leq \frac{r}{\sqrt{2}}$, consider the tiling depicted in the right part of Figure 7 . Discs of this tiling do not cover each other's centers; therefore, any grid covering algorithm that covers all the points of $X$ using only the grid discs depicted in the right part of Figure 7 does not form an unbounded connected component, a.s., for any value of $\lambda$.

For the remaining values of $\delta$, we can use the same tiling of the two cases depicted in Figure 7, scaled by the appropriate factor.

Case 2. In this case, two disc centers are considered connected if and only if the corresponding discs of radius $r$ overlap by a region of measure at least $\varepsilon>0$, where the value of $\varepsilon$ depends on the ratio $\frac{R}{r}$.
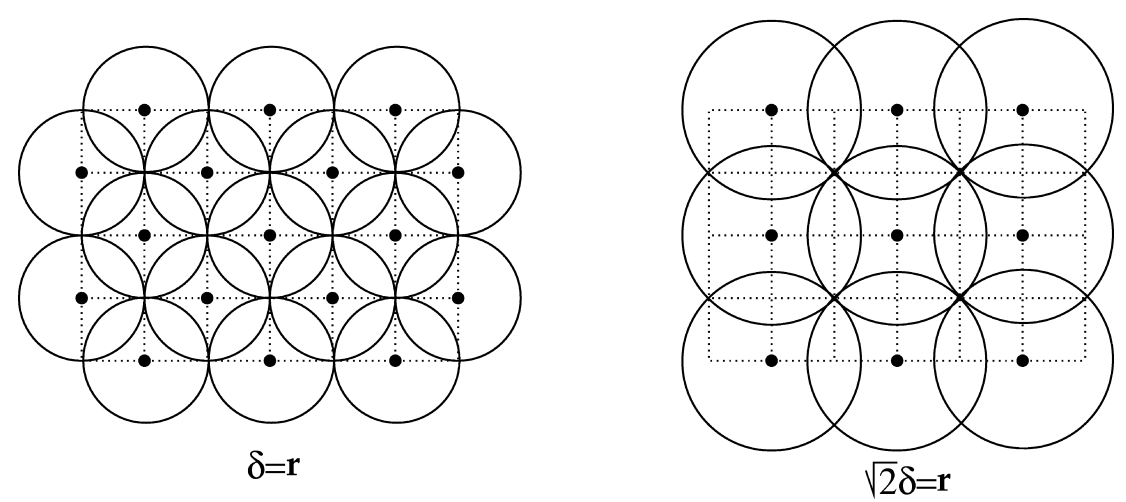

FIG. 7. Theorem 4.1, Case 1: two tilings of the plane by discs centered on a grid that do not cover each other's centers. 


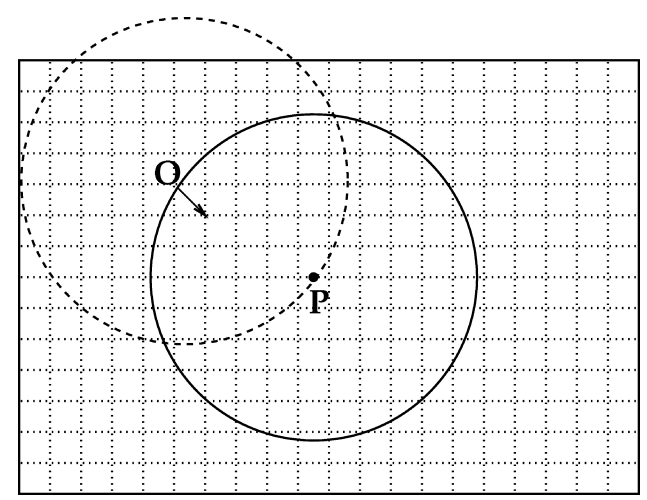

FIG. 8. Theorem 4.1, Case 2: a Poisson point $P$ is covered by a disc centered at point $O$, which is within $r$ from $P$. The covering disc can be moved to a nearby grid vertex, that is inside the solid disc and is within $\sqrt{2} \delta$ from $O$, and still covers point $P$.

We follow a construction similar to that used to prove Proposition 3.4. Draw circles of radii $\{3 k r, k \in \mathbb{N}\}$ around the origin, and notice that a.s. no Poisson point falls on any of these circles. Then cover the Poisson points, each with a disc of radius $r$, without intersecting these circles. Notice that the circles divide the plane into finite annuli, whose boundaries are not covered by discs. We now approximate this covering using a grid covering. Consider a square grid $G$ and move each disc of the above covering to the nearest vertex of $G$ that still allows it to cover its corresponding Poisson point. Note that each disc needs to be translated by at most $\sqrt{2} \delta$. That is because a Poisson point is covered by a disc centered within $r$ from it, and there is always a grid vertex, within radius $r$ from the Poisson point, that is also within $\sqrt{2} \delta$ from this center (see Figure 8).

By this translation, some discs may intersect the boundaries of the annuli that were previously untouched. We then take the grid size $\delta$ so small that any two discs that intersect these boundaries do not overlap by an area of measure greater than or equal to $\varepsilon$ and are therefore not connected. It immediately follows that, for any given value of the density $\lambda$, a.s. there is not any unbounded connected component for this covering.

Case 3. This case is proven by Theorem 3.4.

Case 4. In this case, two disc centers are considered connected if and only if they are at a distance of at most $R$ (see right side of Figure 6). In other words we are interested in the percolation of large discs of radius $R / 2$ around the disc centers. A small disc of radius $\varepsilon=R / 2-r>0$ centered at a point of the Poisson process must be contained in the large disc of radius $R / 2$ around the centre of the disc covering that point. See Figure 9.

Therefore if these small discs percolate, as they will when $\lambda>\lambda(\varepsilon)$, the large discs must also percolate. 


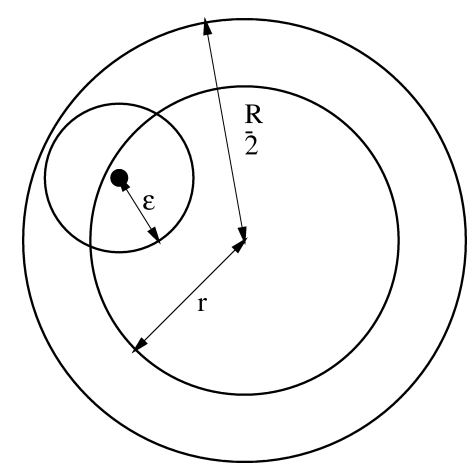

FIG. 9. Case 4: a small discs of radius $\varepsilon=R / 2-r$ around a point of $X$ must lie inside the disc of radius $R / 2$ around the centre of the covering disc.

5. Optimal algorithms. In this section we explore the notion of optimal algorithms, that is, those which use as few discs as possible and show that $n$-square algorithms are asymptotically optimal. The work in this section is philosophically close to that in Yukich (1998).

Fix the density of points, $\lambda$ and $r$, and extend the definition of the density of a covering $\mathcal{A}$ to be

$$
\delta_{\mathcal{A}}=\lim _{n \rightarrow \infty} \frac{\text { number of discs centered in } B_{n}}{n^{2}}
$$

if this exists and is a constant a.s., and $\infty$ otherwise. We then define the optimal density to be

$$
\delta^{\mathrm{opt}}=\inf _{\mathcal{A}} \delta_{\mathcal{A}}
$$

where the infimum runs over all covering algorithms. An optimal algorithm $\mathcal{A}^{\mathrm{opt}}$, is one for which, a.s., $\delta^{\text {opt }}=\delta_{\mathcal{A}^{\text {opt }}}$. We would not expect such an algorithm to have a finite horizon.

First we show that $n$-square algorithms can get as close as we like to the optimal density. Define $\delta_{n}$ to be the density of discs under an $n$-square algorithm. By ergodicity $\delta_{n}<\infty$ exists. Notice that $\delta_{n}$ does not depend upon the particular $n$-square algorithm we choose.

THEOREM 5.1. Given $\varepsilon>0$, there exists $n_{\varepsilon}$ such that $\delta_{n_{\varepsilon}}<\delta^{\mathrm{opt}}+\varepsilon$, and hence $\delta^{\mathrm{opt}}=\inf _{n} \delta_{n}$.

PROOF. We prove this theorem by contradiction, so suppose that we can find an $\varepsilon$ such that there is no $n$-square covering with density between $\delta^{\text {opt }}$ and $\delta^{\text {opt }}+\varepsilon$. We can find another covering, $\hat{\mathcal{A}}$ say, with density $\delta_{\hat{\mathcal{A}}} \in\left[\delta^{\mathrm{opt}}, \delta^{\mathrm{opt}}+\varepsilon / 4\right]$, by the definition of $\delta^{\mathrm{opt}}$. 
Choose $\gamma>0$ such that $(1-\gamma)\left(\delta^{\text {opt }}+\varepsilon / 2\right)+\gamma(1+\varepsilon / 2) / r^{2}<\delta^{\text {opt }}+\varepsilon$. Note that the number of discs necessary to cover an $n$-square is at most $\lceil n / r\rceil^{2}$.

As

$$
\delta_{\hat{\mathcal{A}}}=\lim _{n \rightarrow \infty} \frac{\text { number of discs centered in } B_{n}}{n^{2}} \quad \text { a.s., }
$$

we can choose $n_{\varepsilon}>1$ sufficiently large that (a) the number of discs centered in $B_{n_{\varepsilon}}$ is less than $n_{\varepsilon}^{2}\left(\delta^{\text {opt }}+\varepsilon / 2\right)$ with probability larger than $1-\gamma$, and (b) $\lceil n / r\rceil^{2}(1 / n)^{2} \leq(1+\varepsilon / 2) / r^{2}$.

Given a finite set of points there are a finite number of distinct possible coverings of those points, where we call two coverings distinct if there exist a set of points covered by one disc in one of the coverings but covered by two or more in the other. Coverings that are not distinct are equivalent. We will occasionally work with the equivalence classes of theses coverings.

We now define a covering of the box $B_{n_{\varepsilon}}$ which is based upon $\hat{\mathcal{A}}$ but is independent of the points outside $B_{n_{\varepsilon}}$. Given a point configuration $\pi$ in $B_{n_{\varepsilon}}$ there is a finite set of equivalent classes of coverings of these points. Let $S_{\pi}$ be the subset of equivalence classes which occur with positive probability if we use $\hat{\mathcal{A}}$ to cover $\left.\pi \cup X\right|_{B_{n_{\varepsilon}}^{c}} ^{\prime}$, where $\left.X\right|_{B_{n_{\varepsilon}}^{c}} ^{\prime}$ is a Poisson process on $B_{n_{\varepsilon}}^{c}$ independent of $\pi$ and $X$. In each equivalence class all coverings use the same number of discs so we can choose an equivalence class from $S_{\pi}$ in which the number of discs used is minimal, according to some deterministic rule. Choose a covering from this class, according to some other deterministic rule. This is the covering we use to cover $B_{n_{\varepsilon}}$. Note that this is independent of the actual point process outside $B_{n_{\varepsilon}}$. The expected number of discs required to cover the points in $B_{n_{\varepsilon}}$ under this algorithm can be at most that under $\hat{A}$.

We now divide the plane into squares of size $n_{\varepsilon}$ and cover each square independently using the same algorithm in each as we use on $B_{n_{\varepsilon}}$. For those squares for which this requires at most $n_{\varepsilon}^{2}\left(\delta^{\text {opt }}+\varepsilon / 2\right)$ discs, we use this covering. In the other squares we cover optimally, which means that we have a density of at most $(1+\varepsilon / 2) / r^{2}$ on these squares.

We have created an algorithm that covers each square of size $n_{\varepsilon}$ independently, and which therefore cannot have a density less than $\delta_{n_{\varepsilon}}$. However, the density of the covering is at most $(1-\gamma)\left(\delta^{\text {opt }}+\varepsilon / 2\right)+\gamma(1+\varepsilon / 2) / r^{2}<\delta^{\text {opt }}+\varepsilon$, and we have a contradiction.

Next, we strengthen the previous proposition.

THEOREM 5.2. Let $\delta_{n}$ be the density of discs under an n-square algorithm. Then $\lim _{n \rightarrow \infty} \delta_{n}=\delta^{\mathrm{opt}}$.

PROOF. We know that $\delta^{\text {opt }}=\inf _{n} \delta_{n}$ and that $\delta_{n} n^{2}$ is the expected number of discs needed to cover an $n$-square. For the sake of contradiction suppose that there 


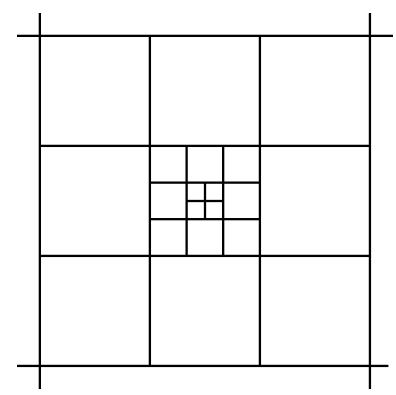

FIG. 10. Division of the plane into squares of increasing size.

exist $\varepsilon>0$ and a sequence $\left\{t_{1}, t_{2}, \ldots\right\}, \lim _{i} t_{i}=\infty$, such that $\delta_{t_{i}}>\delta^{\text {opt }}+\varepsilon$ for all $i$. However, we can choose $\alpha$ so that $\delta_{\alpha}<\delta^{\text {opt }}+\varepsilon / 3$. We can also choose $i$ so large that

$$
\left(1-\left\lfloor\frac{t_{i}}{\alpha}\right\rfloor^{2}\left(\frac{\alpha}{t_{i}}\right)^{2}\right) \lambda<\frac{\varepsilon}{3} .
$$

The reason we need this will become clear shortly.

We cover the square $B_{t_{i}}$ as follows. We first divide as much of the square as possible into squares of size $\alpha$. Each of these we cover optimally. We have an area of $\left(t_{i}^{2}-\alpha^{2}\left\lfloor\frac{t_{i}}{\alpha}\right\rfloor^{2}\right)$ left, and each of the points in this area we cover with one disc. This gives us a covering with expected density

$$
\left\lfloor\frac{t_{i}}{\alpha}\right\rfloor^{2}\left(\frac{\alpha}{t_{i}}\right)^{2} \delta_{\alpha}+\left(1-\left\lfloor\frac{t_{i}}{\alpha}\right\rfloor^{2}\left(\frac{\alpha}{t_{i}}\right)^{2}\right) \lambda \leq \delta^{\mathrm{opt}}+\frac{\varepsilon}{3}+\frac{\varepsilon}{3} .
$$

However, the minimal expected density for any algorithm covering the box $B_{t_{i}}$, $\delta_{t_{i}}>\delta^{\mathrm{opt}}+\varepsilon$, so we have a contradiction.

Note that it is still not clear a priori that an optimal algorithm should exist. The existence of an optimal density, defined as the infimum over all attainable densities, does not have to be attainable itself. However, we have the following proposition:

\section{PROPOSITION 5.3. There exists an optimal algorithm.}

Proof. Divide the plane into squares of increasing size, as in Figure 10. Cover the points in each of the squares in some optimal way. This gives a covering of the plane and we claim that it is optimal. To see this choose some $\varepsilon>0$ and notice that by Theorem 5.2 we can find $n=2^{m}$ so large that $\delta_{n}<\delta^{\text {opt }}+\varepsilon$. The fraction of the plane covered by squares of side length smaller than $n$ is 0 , so our algorithm has density at most $\delta^{\mathrm{opt}}+\varepsilon$. However, $\varepsilon$ was arbitrary, and therefore our algorithm must be optimal. 
6. Open problems. Now we mention a number of open problems:

1. For which classes of algorithms does there exist a critical density? By this we mean a critical value $\lambda_{c}$, such that percolation occurs for $\lambda>\lambda_{c}$ and does not occur for $\lambda<\lambda_{c}$.

2. For which classes of algorithms is the infinite cluster unique? In other words, when do we have either 0 or 1 infinite cluster, a.s.?

3. We have shown in Theorem 3.6 that if we have an algorithm with a finite horizon, which is shift invariant under two linearly independent shifts and has a bounded density of discs, then we must have percolation for $\lambda$ high enough. We have also shown in Theorem 3.8 that we can have a completely shift invariant covering algorithm with an unbounded density of discs and no finite horizon that does not percolate, even for high values of $\lambda$. Do we have percolation for $\lambda$ high enough for a finite horizon algorithm invariant under a pair of shifts, with an unbounded density of discs? Do we necessarily have percolation for $\lambda$ high enough if we have a bounded density of discs and shift invariance but no finite horizon?

Acknowledgments. The authors thank Richard Gill. We also thank Johan Segers for reading an early version of this article very carefully and for the many interesting comments and observations that resulted. Massimo Franceschetti thanks his fellow student Matthew Cook for many interesting discussions and for reading and providing feedback on some of the proofs. We also thank the referee for scrutinizing the paper scrupulously and suggesting some improved proofs.

\section{REFERENCES}

Agarwal, P. J. and Sharir, M. (1998). Efficient algorithms for geometric optimization. ACM Computing Surveys $30412-458$.

Booth, L. (2002). Random spatial structures and sums. Ph.D. dissertation, Utrecht Univ.

Booth, L., Bruck, J., Franceschetti, M. and Meester, R. (2002). Technical Report ETR037, Caltech. Available at www.paradise.caltech.edu/ETR.html.

Durrett, R. and Griffeath, D. (1983). Supercritical contact processes on $\mathbb{Z}$. Ann. Probab. 11 $1-15$.

ERDős, P. and RÉnYi, A. (1959). On random graphs, I. Publ. Math. Debrecen. 6 290-297.

ERDős, P. and RÉNYI, A. (1960). On the evolution of random graphs. Publ. Math. Inst. Hungarian Acad. Sci. 5 17-61.

ERDős, P. and RÉNYI, A. (1961a). On the evolution of random graphs. Bull. Internat. Inst. Statist. Tokyo 38 343-347.

ERDős, P. and RÉNYI, A. (1961b). On the strength of connectedness of a random graph. Acta Math. Acad. Sci. Hungar. 12 261-267.

Franceschetti, M., Cook, M. and Bruck, J. (2001). A geometric theorem for approximate disk covering algorithms. Available at www.paradise.caltech.edu/ETR.html.

Gerla, M. and TsAi, J. T. (1995). Multicluster, mobile, multimedia radio network. ACM/Baltzer Wireless Networks 1 255-265.

Gilbert, E. N. (1961). Random plane networks. SIAM J. 9 533-543. 
Gupta, P. and Kumar, P. R. (1998). Critical power for asymptotic connectivity in wireless networks. In Stochastic Analysis, Control, Optimization and Applications: A Volume in Honor of W. H. Fleming (W. M. McEneany, G. Yin and Q. Zhang, eds.) 547-566. Birkhäuser, Boston.

Gupta, P. and KumAR, P. R. (2000). The capacity of wireless networks. IEEE Trans. Inform. Theory 46 388-404.

Hall, P. (1985). On continuum percolation. Ann. Probab. 13 1250-1266.

HALL, P. (1988). Introduction to the Theory of Coverage Processes. Wiley, New York.

MEESTER, R. and RoY, R. (1994). Uniqueness of unbounded occupied and vacant components in boolean models. Ann. Appl. Probab. 4 933-951.

Meester, R. and Roy, R. (1996). Continuum Percolation. Cambridge Univ. Press.

Menshikov, M. V. (1986). Coincidence of critical points in percolation problems. Soviet Math. Dokl. 24 856-859.

Penrose, M. (1997). The longest edge of the random minimal spanning tree. Ann. Appl. Probab. 7 340-361.

RoY, R. (1990). The Russo-Seymour-Welsh theorem and the equality of the critical densities and critical dual densities for continuum percolation on $\mathbb{R}^{2}$. Ann. Probab. 18 1563-1575.

Yukich, J. E. (1998). Probability Theory of Classical Euclidean Optimization Problems. Lecture Notes in Math. 1675. Springer, New York.

L. ВоотH

MATHEMATICAL INSTITUTE

UTRECHT UNIVERSITY

PO BOX 80010

3508TA UTRECHT

THE NETHERLANDS

E-MAIL: booth@math.uu.nl
J. BRUCK

M. FRANCESCHETTI

MOORE LABORATORY

OF ELECTRICAL ENGINEERING

CALIFORNiA InSTITUTE OF TECHNOLOGY

M.C. 136-93

PASAdEna, CALIFornia 91125

E-MAIL: bruck@paradise.caltech.edu massimo@paradise.caltech.edu

R. MEESTER

FACULTY OF EXACT SCIENCES

FREE UNIVERSITY OF AMSTERDAM

DE BoelelaAn 1083A

1081 HV AMSTERDAM

THE NETHERLANDS

E-MAIL: rmeester@cs.vu.nl 